\title{
Mycobacterium avium subsp. paratuberculosis (MAP) infection in the endangered huemul deer (Hippocamelus bisulcus) in Patagonia
}

\author{
Paulo Corti ${ }^{\mathrm{a}}$, Bernardita Collado ${ }^{\mathrm{a}, \mathrm{b}, \mathrm{c}}$, Carlos Riquelme $^{\mathrm{d}}$, \\ Camilo Tomckowiack ${ }^{b, c}$, Miguel Salgado ${ }^{b^{*}}$
}

\begin{abstract}
In a huemul (Hippocamelus bisulcus) population sympatric with cattle, we found evidence of Mycobacterium avium subsp. paratuberculosis (MAP) infection. Three huemul faecal pellet samples and two cows pats were collected and cultured for MAP presence. DNA was then extracted for PCR analysis of all signal-positive cultures. To assess whether MAP isolates obtained from huemul faeces were associated with typical MAP isolated from livestock, positive confirmed culture samples were sub-typed using a combination of five Mycobacterial Interspersed Repetitive Unit-Variable Number Tandem Repeat Analysis and one Short Sequence Repeat analysis markers. All faecal samples from both species were MAP positive. One huemul presented a different bacteria profile genotype not described before, suggesting that huemul and cattle in Patagonia could carry a unique MAP strain.

Key words: Patagonia, huemul, cattle, spillover, paratuberculosis, typing.
\end{abstract}

\section{INTRODUCTION}

The International Union for the Conservation of Nature estimates that over $24 \%$ of the world's extant mammals are currently threatened with extinction, yet infectious diseases have only been listed as a major threat for a small fraction $(1.1 \%)^{1}$. It is likely that diseases are underrepresented as a contributing threat to wildlife extinction, especially given that less than half $(39 \%)$ of critically endangered and endangered artiodactyls, carnivores, and primates from the 2006 IUCN Red List have any published records of pathogens from their wild populations (Pedersen et al 2007). Recently, evidence linking huemul (Hippocamelus bisulcus), an endemic and endangered deer species from southern Chile and Argentina (Corti et al 2010), with Mycobacterium avium subsp. paratuberculosis (MAP) infection has been reported (Salgado et al 2017). MAP is the causative agent of Johne's disease, infection mostly affecting domestic ruminants, but it has been shown to pose a threat also to wildlife (Manning and Collins 2001, Salgado et al 2015). However, the pathogen has also been

Received: 17.07.2019.

Accepted: 22.10.2019.

aLaboratorio de Manejo y Conservación de Vida Silvestre, Instituto de Ciencia Animal y Programa de Investigación Aplicada en Fauna Silvestre, Facultad de Ciencias Veterinarias, Universidad Austral de Chile, Valdivia, Chile.

bInstituto de Medicina Preventiva Veterinaria, Facultad de Ciencias Veterinarias, Universidad Austral de Chile, Valdivia, Chile.

cEscuela de Graduados, Facultad de Ciencias Veterinarias, Universidad Austral de Chile, Valdivia, Chile.

${ }^{d}$ Escuela de Graduados, Facultad de Ciencias; Universidad Austral de Chile, Valdivia, Chile.

*Corresponding author: M Salgado; miguelsalgado@uach.cl

1 IUCN, International Union for the Conservation of Nature. 2018. IUCN red list of threatened species. Available at http://www.redlist. org isolated from non-ruminants species and even from humans, associating it with Crohn's disease (Chiodini et al 2012).

Although huemul inhabits remote areas with limited contact with domestic animals/livestock and exists at low population density, the presence of pathogens like MAP may be interpreted as an indicator of spill-over infections from domestic animals. Then, to improve our knowledge about this current issue in huemul, a cross-sectional study was conducted on few huemul and cattle co-inhabiting the same area to inquire possible epidemiological interactions at Torres del Paine National Park in Chilean Patagonia (figure 1).

\section{MATERIAL AND METHODS}

The sampling was carried out in November 2016, at the end of the southern hemisphere spring. Three huemul faecal pellet samples, each containing from six to 10 droppings, and two cows pats were collected (Figure 1). The sampling selection criteria was that faecal material from both species was no more than one-day old, estimated according to the appearance of deer pellets and the characteristics of cow pats (Lehmkuhl et al 1994). To collect the faeces, we used sterile latex gloves avoiding soil contamination, and the samples were individually kept in sealed plastic bags previously labelled. Samples were stored one week under a refrigeration temperature of $5{ }^{\circ} \mathrm{C}$ until laboratory analyses. Faecal samples were processed in a liquid culture system (BACTEC-MGIT 960, Sparks, Maryland 21152, USA) for MAP presence according to the manufacturer's protocol, and then DNA was extracted from all signal-positive cultures. Real-time IS900 PCR was performed for MAP detection verification to confirm positive samples (Salgado et al 2014). The MAP confirmation consisted of a PCR system targeting the insertion element IS900. The PCR total reaction was $20 \mu \mathrm{l}$, from which $5 \mu \mathrm{l}$ was DNA template, $10 \mu \mathrm{l}$ were 23 TaqMan Universal MasterMix (Roche Applied Science, 


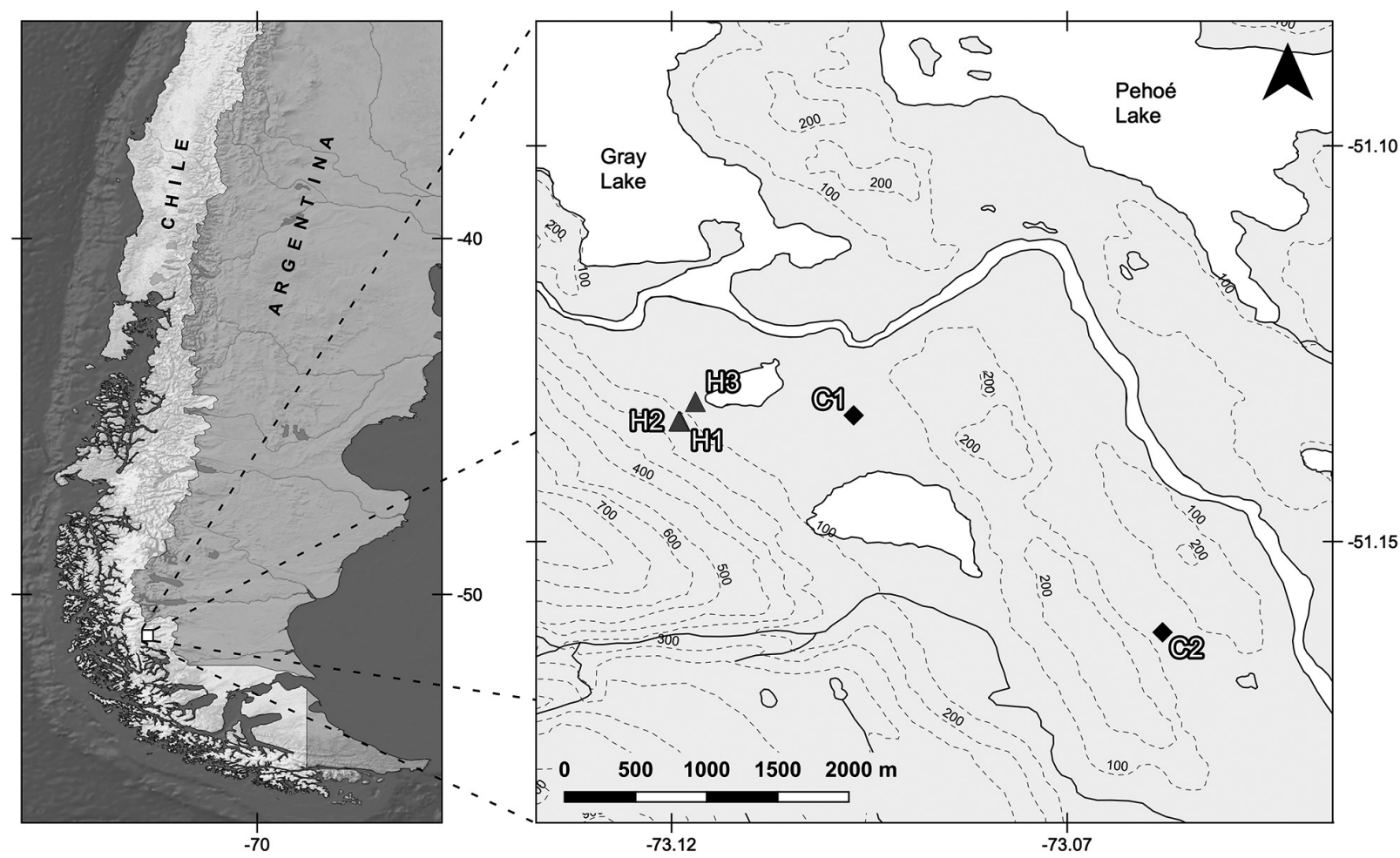

Figure 1. Geographic location of the study area and collection sites at Torres del Paine National Park in Chilean Patagonia. Black dots indicate environmental huemul $(\mathrm{H})$ and cow $(\mathrm{C})$ faecal samples.

Indianapolis, Indiana 46250, USA), $2 \mu \mathrm{l}$ of each primer $(0.2 \mu \mathrm{M})$, and $1 \mu \mathrm{l}$ probe $(0.1 \mu \mathrm{M})$. The sequence for IS900 primers, which amplified a 63 nucleotide fragment of the IS900 gene target, was 5' GACGCGATGATCGAGGAG-39 (L) and 59-GGGCATGCTCAGGATGAT-3' (R). The reactions were run in a Roche LightCycler 2.0 system (Roche Applied Science) under the following standard conditions: one cycle to $95^{\circ} \mathrm{C}$ for $10 \mathrm{~min}, 45$ cycles with three steps of $95^{\circ} \mathrm{C}$ for $10 \mathrm{sec}, 60^{\circ} \mathrm{C}$ for $30 \mathrm{sec}, 72^{\circ} \mathrm{C}$ for $1 \mathrm{sec}$, and a final cooling step at $40^{\circ} \mathrm{C}$ for $30 \mathrm{sec}$. Negative and positive (MAP ATCC 19698) PCR controls were included as well as a DNA extraction negative and positive control.

To assess whether MAP isolates obtained from huemul faeces were associated with typical MAP isolated from livestock, positive confirmed culture samples were sub-typed using a combination of five Mycobacterial Interspersed Repetitive Unit-Variable Number Tandem Repeat Analysis (MIRU-VNTR 292, 25, X3, 7, and 3; Thibault et al 2007), and one Short Sequence Repeat analysis markers (SSR 2; Amonsin et al 2004). The combination of MIRU-VNTR and SSR allele repeats formed a unique profile that allowed the allele profile to be compared to MAP sub-types obtained from a survey on huemul and other domestic animals in Chile (Salgado et al 2017).

\section{RESULTS AND DISCUSSION}

All three huemul faecal samples were culture positive for MAP, and the two cow faeces samples were also positive for MAP, both confirmed through PCR (table 1). All DNA

Table 1. Huemul MIRU-VNTR and SSR pattern result and its comparison with local cow strains from Torres del Paine National Park.

\begin{tabular}{lccccccccc}
\hline & Sample ID & 292 & $\mathrm{x} 3$ & 25 & 47 & 3 & MIRU-VNTR* & INMV** & SSR-L2 $* * *$ \\
\hline HUEMUL & 134 & 4 & 2 & 3 & 3 & 2 & 42332 & INMV 1 & 10 \\
HUEMUL & 135 & 4 & 2 & 3 & 3 & 2 & 42332 & INMV 1 & 10 \\
HUEMUL & 137 & 3 & 2 & 3 & 3 & 2 & 32332 & INMV 2 & 14 \\
COW & 138 & 3 & 2 & 3 & 3 & 2 & 32332 & INMV 2 & 14 \\
\hline
\end{tabular}

*MIRU-VNTR: Mycobacterial Interspersed Repetitive Unit-Variable Number Tandem Repeat Analysis. **INMV: The INRA Nouzilly MIRU-VNTR profile nomenclature, as defined by Thibault et al (2007).

***SSR L2: Short Sequence Repeat analysis markers-Locus 2. 
of the isolated strains showed a sufficient quality to consider the results of the molecular characterisation conclusive, except for the strain obtained from one of the cows. The lack of variation between one cow and one huemul MAP strains suggests that cattle and huemul populations are sympatric, as previously stated (Salgado et al 2017). This result suggests that both species share the same bacteria, evidencing interspecies transmission as shown elsewhere between wildlife and livestock based on the same typing protocol (Fritsch et al 2012). Also, Thibault et al (2008) highlighted that MIRU-VNTR typing followed by SSR was the most cost-effective and efficient strategy for strain discrimination.

It has been also hypothesised (Salgado et al 2017) that the infection to huemul primarily may be through drinking faecal-contaminated water and secondarily through faecal-oral route since huemul species are browsers, mostly feeding on shrubs, trees, and forbs but rarely on graminoids near the ground (Vila et al 2009). However, one specific MIRU-VNTR and SSR genotype found in Torres del Paine National Park, from huemul faecal material, was different from those detected in cattle herds and from genotypes previously informed in Chile (table 1)(Salgado et al 2017).

It is highly interesting, within this restricted area, that epidemiologically associated free-ranging cattle and huemul could carry specific MAP genotypes. Therefore large-scale studies are urgently needed to gain deeper knowledge on this important sanitary issue in an endangered species like the huemul.

\section{REFERENCES}

Amonsin A, Li LL, Zhang Q, Bannantine JP, Motiwala AS, et al. 2004. Multilocus short sequence repeat sequencing approach for differentiating among Mycobacterium avium subsp. paratuberculosis strains. J Clin Microbiol 42, 1694-1702.
Chiodini RJ, Chamberlin WM, Sarosiek J, McCallum RW. 2012. Crohn's disease and the mycobacterioses: a quarter century later: causation or simple association? Crit Rev Microbiol 38, 52-93.

Corti P, Wittmer HU, Festa-Bianchet M. 2010. Dynamics of a small population of endangered huemul deer (Hippocamelus bisulcus) in Chilean Patagonia. J Mammal 91, 690-697.

Fritsch I, Luyven G, Köhler H, Lutz W, Möbius P. 2012. Suspicion of Mycobacterium avium subsp. paratuberculosis transmission between cattle and wild-living red deer (Cervus elaphus) by multitarget genotyping. Appl Environ Microbiol 78, 1132-1139.

Lehmkuhl JF, Hansen CA, Sloan K. 1994. Elk pellet-group decomposition and detectability in coastal forests of Washington. $J$ Wildl Manage 58, 664-669.

Manning EJ, Collins MT. 2001. Mycobacterium avium subsp. paratuberculosis: pathogen, pathogenesis and diagnosis. Rev Sci Tech Int Off Epi 20, 33-150.

Pedersen AB, Jones KE, Nunn CL, Altizer SA. 2007. Infectious disease and mammalian extinction risk. Conserv Biol 21, 1269-1279.

Salgado M, Verdugo C, Heuer C, Castillo P, Zamorano P. 2014. A novel low-cost method for Mycobacterium avium subsp. paratuberculosis DNA extraction from an automated broth culture system for real-time PCR analysis. $J$ Vet Sci 15, 233-239.

Salgado M, Aleuy OA, Sevilla IA, Troncoso E. 2015. Detection of Mycobacterium avium subsp. paratuberculosis in a cattle/pudu interface. Arq Bras Med Vet Zootec 67, 1205-1209.

Salgado M, Corti P, Verdugo C, Tomckowiack C, Moreira R, et al. 2017. Evidence of Mycobacterium avium subsp. paratuberculosis (MAP) infection in huemul deer (Hippocamelus bisulcus) in Patagonian fjords. Austral J Vet Sci 49, 135-137.

Thibault VC, Grayon M, Boschiroli ML, Hubbans C, Overduin P, et al 2007. New variable-number tandem-repeat markers for typing Mycobacterium avium subsp. paratuberculosis and M. avium strains: comparison with IS900 and IS1245 restriction fragment length polymorphism typing. J Clin Microbiol 45, 2404-2410.

Thibault VC, Grayon M, Boschiroli ML, Willery E, Allix-Béguec C, et al. 2008. Combined multilocus short-sequence-repeat and mycobacterial interspersed repetitive unit-variable-number tándem repeat typing of Mycobacterium avium subsp. paratuberculosis isolates. J Clin Microbiol 46, 4091-4094.

Vila AR, Galende GI, Pastore H. 2009. Feeding ecology of the endangered huemul (Hippocamelus bisulcus) in Los Alerces National Park, Argentina. Mastozool Neotrop 16, 423-431. 
